\title{
Neurodegeneration Triggers Peripheral Immune Cell Recruitment into the Forebrain
}

\author{
Miriam Scheld, ${ }^{1 \star}$ Bernhard Josef Rüther, ${ }^{1 \star}$ René Große-Veldmann, ${ }^{1}$ Kim Ohl, ${ }^{2}$ Klaus Tenbrock, ${ }^{2}$ Daniela Dreymüller, ${ }^{3}$ \\ Petra Fallier-Becker, ${ }^{4}$ Adib Zendedel, ${ }^{1}$ Cordian Beyer, ${ }^{1}$ Tim Clarner,${ }^{1 \dagger}$ and Markus Kipp ${ }^{\dagger \dagger}$ \\ ${ }^{1}$ Institute of Neuroanatomy, ${ }^{2}$ Department of Pediatrics, and ${ }^{3}$ Institute of Pharmacology and Toxicology, Faculty of Medicine, RWTH Aachen University, \\ D-52074 Aachen, Germany, ${ }^{4}$ Institute of Pathology and Neuropathology, University of Tuebingen, D-72076 Tuebingen, Germany, and ${ }^{5}$ Department of \\ Anatomy II, Ludwig Maximilians University of Munich, D-80336 Munich, Germany
}

Brain-intrinsic degenerative cascades have been proposed to be an initial factor driving lesion formation in multiple sclerosis (MS). Here, we identify neurodegeneration as a potent trigger for peripheral immune cell recruitment into the mouse forebrain. Female $\mathrm{C} 57 \mathrm{BL} / 6 \mathrm{mice}$ were fed cuprizone for 3 weeks, followed by a period of 2 weeks on normal chow to induce the formation of lesion foci in the forebrain. Subsequent immunization with myelin oligodendrocyte glycoprotein 35-55 peptide, which induces myelin autoreactive T cells in the periphery, resulted in massive immune cell recruitment into the affected forebrain. Additional adoptive transfer experiments together with flow cytometry analysis underline the importance of brain-derived signals for immune cell recruitment. This study clearly illustrates the significance of brain-intrinsic degenerative cascades for immune cell recruitment and MS lesion formation. Additional studies have to address the signaling cascades and mechanistic processes that form the top-down communication between the affected brain area, neurovascular unit, and peripheral immune cells.

Key words: cytodegeneration; invasion; trigger

Significance Statement

We identify neurodegeneration as a potent trigger for peripheral immune cell recruitment into the forebrain. Thus, immune cell recruitment might be a second step during the formation of new inflammatory lesions in multiple sclerosis. A better understanding of factors regulating neurodegeneration-induced immune cell recruitment will pave the way for the development of novel therapeutic treatment strategies.

\section{Introduction}

Histopathological characteristics of multiple sclerosis (MS) lesions include oligodendrocyte death, demyelination, gliosis, axonal damage, and peripheral immune cell invasion. However, it is not entirely clear which mechanisms regulate the development of new inflammatory brain lesions. Although some reports suggest a key role for adaptive immunity during the earliest stages of lesion genesis (Lucchinetti et al., 2000; Maggi et al., 2014), others have

Received June 27, 2015; revised Dec. 16, 2015; accepted Dec. 18, 2015.

Author contributions: C.B., T.C., and M.K. designed research;M.S., B.J.R., R.G.-V., K.O., K.T., D.D.,P.F.-B., A.Z., and T.C. performed research; M.S., B.J.R., R.G.-V., K.O., D.D., P.F.-B., A.Z., T.C., and M.K. analyzed data; B.J.R., C.B., T.C., and M.K. wrote the paper.

This study was supported by the Dr. Robert Pfleger Stiftung (M.K.). The technical support from P. Ibold and H. Helten is acknowledged.

The authors declare no competing financial interests.

${ }^{*} M$.S. and B.J.R. contributed equally to this work as first authors.

${ }^{\dagger}$ T.C. and M.K. contributed equally to this work as last authors.

Correspondence should be addressed to Markus Kipp, Anatomische Anstalt, Lehrstuhl II-Neuroanatomie, Pettenkoferstrasse 11, D-80336 Munich, Germany. E-mail: markus.kipp@med.uni-muenchen.de.

DOI:10.1523/JNEUROSCI.2456-15.2016

Copyright $\odot 2016$ the authors $\quad 0270-6474 / 16 / 361410-06 \$ 15.00 / 0$ proposed that neurodegenerative events are the initial factors driving MS lesion formation, including oligodendrocyte death, focal microglia activation, and axonal damage (Barnett and Prineas, 2004). This series of events was recently termed "insideoutside model” (Stys et al., 2012). Locatelli et al. (2012) investigated the initiation of MS-like pathology in a mouse model in which the diphtheria toxin receptor is specifically expressed in oligodendrocytes. Despite diphtheria toxin-induced neurodegeneration, even conditions favoring autoimmunity-bystander activation, removal of regulatory $\mathrm{T}\left(\mathrm{T}_{\text {reg }}\right)$ cells, and application of demyelinating antibodies - did not result in peripheral immune cell formation. The authors concluded that oligodendrocyte death with concomitant reactive gliosis is not sufficient to trigger CNS inflammation. However, it is not clear whether a neurodegenerative process represents an important catalyst for immune cell recruitment from the "outside" compartment if encephalitogenic $\mathrm{T}$ cells are present there in a relevant number. Several studies have reported the dissociation between inflammation-driven relapses and clinical progression in MS (Kremenchutzky et al., 2006; Scalfari et al., 2010). Furthermore, dissociation between 
therapeutic effects on relapse frequency versus effects on progression of disability became clear in clinical trials (Coles et al., 1999; Rice et al., 2000). Therefore, a better understanding of the interplay of both variables is needed. Recently published data suggest that neurodegeneration indeed can trigger immune cell recruitment into the brain parenchyma (Baxi et al., 2015).

To further test this hypothesis, we combined a classical "neurodegenerative" and a classical autoimmune MS animal model. In the cuprizone (Cup) model, rodents are fed the copper chelator Cup, which results in varying degrees of oligodendroglial damage, demyelination gliosis, and axonal destruction (i.e., neurodegeneration) in the CNS with little, if any, autoimmune reaction (Kipp et al., 2009). Neurodegenerative foci are found widespread within the brain, including distinct forebrain areas such as the hippocampus, corpus callosum, and cortex. In active experimental autoimmune encephalomyelitis (EAE), sensitization to distinct myelin antigens, such as myelin oligodendrocyte glycoprotein 35-55 (MOG) peptide, results in immune cell recruitment into the CNS with subsequent autoimmune-driven inflammation and demyelination. Remarkably, lesions in MOGimmunized animals predominantly arise in the spinal cord and cerebellum.

Here, we demonstrate that peripheral immune cells do not invade the forebrain in MOG-induced EAE under standard conditions. However, severe forebrain inflammation is evident if animals are pre-intoxicated with Cup. Adoptive transfer (AT) experiments together with flow cytometry analysis underline the importance of brain-derived signals for the observed immune cell recruitment. Together, we provide clear evidence that the degeneration caused by Cup treatment acts as a trigger for immune cells to enter the forebrain.

\section{Materials and Methods}

Animals. C57BL/6 female mice (Janvier) were used. Food and water were available ad libitum. All experimental procedures were approved by the Review Board for the Care of Animal Subjects of the district government.

Experimental induction of neurodegeneration and autoreactive T-cell formation. In general, to induce neurodegeneration of the forebrain, mice received a diet containing $0.25 \%$ Cup (Sigma-Aldrich) for 3 weeks mixed into a ground standard rodent chow, followed by a period of 2 weeks on normal chow to allow autonomous lesion progression (Doan et al., 2013). Animals were then assigned randomly to experimental groups. Histopathological stainings of such lesions were already published previously (Grosse-Veldmann et al., 2015). The formation of myelin autoreactive $\mathrm{T}$ cells was achieved by immunization with an emulsion of MOG dissolved in complete Freund's adjuvant (CFA), followed by administration of pertussis toxin (PTX) in PBS, first on the day of immunization and then again the following day (i.e., active EAE). A ready-to-use kit provided by Hooke Laboratories was used. Scoring of disease severity was performed as published previously (Kipp et al., 2011). Mice that did not present with clinical scores were not included in subsequent analyses $(n=4)$.

AT experiments. For classical AT experiments, female C57BL/6 donor mice were immunized as described above and used as a source of encephalitogenic $\mathrm{T}$ cells. Once donor mice developed a clinical response to MOG ( $\sim 11 \mathrm{~d}$ after active immunization), they were killed, and their spleens harvested. To induce in vitro clonal expansion of MOG-reactive lymphocytes, cells were cultured in the presence of MOG (20 $\mu$ g peptide/ $\mathrm{ml}$; Hooke Laboratories), IL12 peptide ( $20 \mathrm{ng} / \mathrm{ml}$; Peprotech), and antimouse IFN $\gamma$ antibodies ( $10 \mu \mathrm{g} / \mathrm{ml}$; Peprotech) for $72 \mathrm{~h}$. Subsequently, 20-30 million cells were injected via the tail vein into mice with or without previous Cup-induced neurodegeneration (see above). In additional experiments, spleens were harvested from Cup-treated animals, processed and cultured as described above, and injected via the tail vein into mice with or without previous Cup-induced neurodegeneration (see Fig. 2C; Cup-AT in co and Cup-AT in cup). This part of the experiment was designed to investigate whether autoreactive T cells are generated in Cup-exposed animals.

Histological, immunohistological, and mRNA-level evaluation. Tissue sampling and processing were performed following established protocols (Clarner et al., 2015). Before paraffin embedding, spinal cord tissues were incubated in EDTA solution for $48 \mathrm{~h}$ at $37^{\circ} \mathrm{C}$. For the quantification of perivascular infiltrates within the forebrain, three H\&E-stained brain sections at the levels 215,245 , and 265 according to the mouse brain atlas by Sidman et al. (http://www.hms.harvard.edu/research/brain/atlas. html) were evaluated by two blinded observers, and the results were averaged. Subsequent slides were evaluated for the quantity of parenchymal cluster of differentiation 3-positive $\left(\mathrm{CD}^{+}\right)$cells in the white matter tract corpus callosum if appropriate. Cell quantification approaches have been performed as published recently (Slowik et al., 2015). Gene expression analyses were performed as published previously (Clarner et al., 2015). To analyze the presence of lipids in cervical lymph nodes of Cuptreated animals, OilRedO (ORO) stains of cryosections were performed using a ready-to-use kit (Abcam) following the instructions of the manufacturer.

Flow cytometry analysis. For cell surface staining, single-cell suspensions were prepared from spleens or cervical lymph nodes and stained with the following antibodies: anti-CD3-Allophycocyanin (APC) (eBioscience), anti-CD4-Pacific Blue (PB; eBioscience), anti-CD25-Phycoerythrin (PE) (eBioscience), anti-Gr-1-PE (eBioscience), and antiCD11b-PB (eBioscience). Intracellular staining of FoxP-FITC (eBioscience) was performed using eBioscience FoxP3 staining buffer kit, according to the instructions of the manufacturer. For measurement of intracellular IL17 production, cells were treated with PMA (20 nM), ionomycin $(2 \mu \mathrm{M})$ (both from Sigma-Aldrich), and GolgiPlug (BD Bioscience) for $5 \mathrm{~h}$, and intracellular staining was performed with anti-IL17Alexa Fluor 488 (eBioscience) using eBioscience FoxP3 staining buffer kit. Samples were analyzed using a FACS Canto II (BD Biosciences), and data were analyzed using FCS Express (De Novo Software) software.

Statistical analysis. For comparative clinical scoring, 39 animals were assigned to the MOG EAE group and 31 to the Cup/MOG group. In each group, two animals did not show clinical signs and were therefore excluded. If not stated otherwise, at least two independent experiments were performed for each experimental group. All data are given as arithmetic means \pm SEMs. Differences between groups were statistically tested by Student's $t$ test using GraphPad Prism 5 (GraphPad Software). $p$ values $<0.05$ were considered to be statistically significant. The following symbols were used to indicate the level of significance: ${ }^{*} p \leq 0.05$, ${ }^{* * *} p \leq 0.001 . \mathrm{ns}$ indicates not significant.

\section{Results}

First, animals were fed Cup to induce forebrain neurodegeneration and were then immunized with MOG peptide (we refer to this experimental group as Cup-MOG). In contrast to a previous report (Maña et al., 2009), the severity of clinical symptoms in classical MOG-immunized EAE mice was comparable in animals with or without previous Cup intoxication. In particular, no significant difference was observed for the parameters disease onset (EAE, $11.24 \pm 0.47$ vs Cup-MOG, $10.56 \pm 0.33 \mathrm{~d}$ after immunization; $p=0.23$ ) and maximum disease score (EAE, $2.31 \pm 0.19$ vs Cup-MOG, $2.3 \pm 0.18 ; p=0.94)$. In line with this observation, inflammatory infiltrates were found in the spinal cord of both groups to a comparable extent (Fig. 1A). Such infiltrates were not present in the spinal cord of control (co) and Cup-fed animals. In a next step, we analyzed the forebrain at distinct rostral-to-caudal levels for the presence of perivascular infiltrates, a hallmark of active MS lesions (Maggi et al., 2014). Although such perivascular infiltrates were virtually absent in the forebrain of co animals and Cup and MOG-immunized animals, the forebrains of CupMOG animals contained a significant number of perivascular infiltrates (Fig. 1B). Such infiltrates were found widespread within the forebrain, including the cortex, corpus callosum, and subcortical regions (Fig. 1C). Remarkably, the number of 

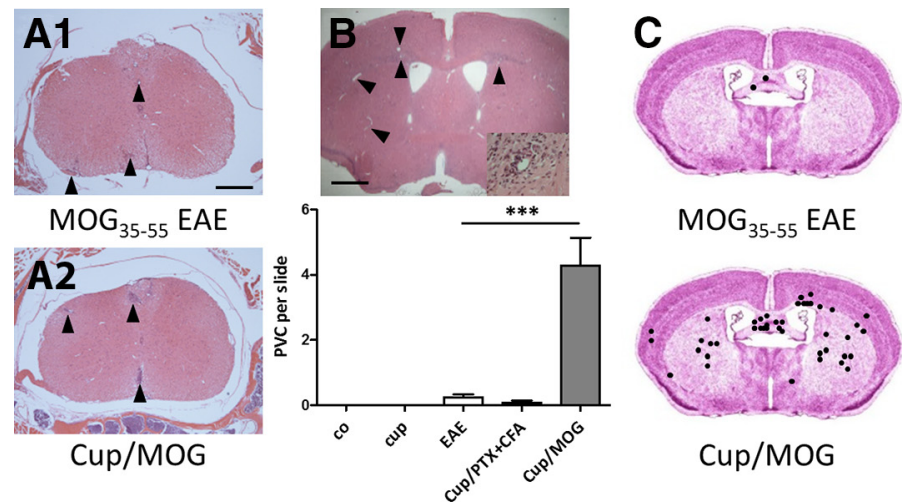

$\mathrm{MOG}_{35-55} \mathrm{EAE}$

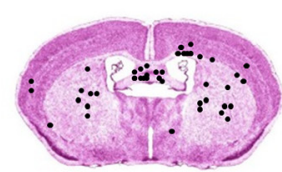

Cup/MOG
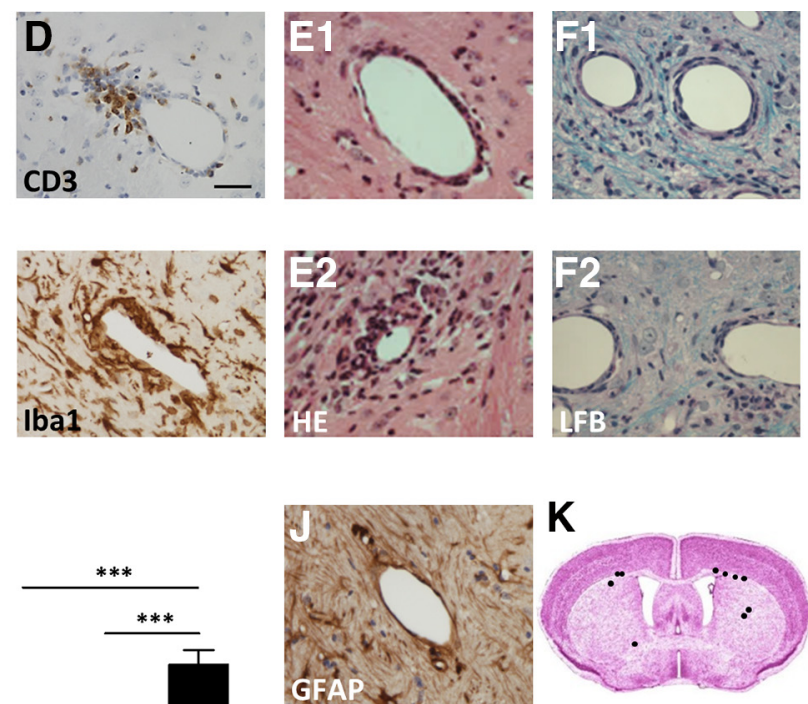

$\mathbf{K}$
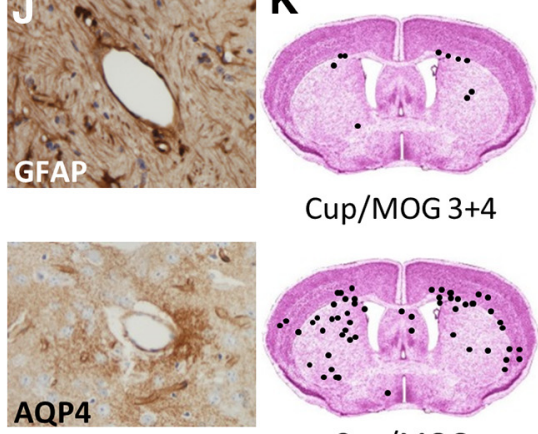

Cup/MOG

Figure 1. A, Shows representative H\&E-stained sections of the spinal cord (at the peak of the disease, $\sim 2$ weeks after immunization). Arrowheads highlight inflammatory foci. $\boldsymbol{B}$ and $\boldsymbol{C}$ show the number and spatial distribution of perivascular infiltrates in the different treatment groups (H\&Estaining). D, Composition of perivascular infiltrates. Both CD ${ }^{+}$lymphocytes and lba $1{ }^{+}$monocytes can be found. $\boldsymbol{E}$ shows two distinct lesion types: $\boldsymbol{E 1}$, perivascular lesions in which immune cells are trapped in the enlarged perivascular space; $\boldsymbol{E 2}$, where immune cells invaded the neuropil. Immune cell neuropil invasion induces demyelination [Luxol fast blue (LFB)/periodic acid Schiff; $F \mathbf{F 1}, \mathbf{F 2}$ ], the breakdown of the glia limitans perivascularis (anti-Glial fibrillary acidic protein; G1, G2, see arrows), and astrocyte polarity loss (anti-AQP4; H1, H2). I, Quantification of $C D 3^{+}$cells into the neuropil. Arrow points at a $C D 3^{+}$lymphocyte within the neuropil. $J$ shows the integrity of the glia limitans perivascularis 6 weeks after immunization. Anti-Glial fibrillary acidic protein presents as a continuous layer of astrocyte end feet around vessels; focal loss of anti AQP-4 stain is not evident anymore. $\boldsymbol{K}$ shows the cumulative number and spatial distribution of perivascular infiltrates after delayed MOG immunization (i.e., 4 instead of 2 weeks after Cup-induced neurodegeneration).

perivascular infiltrates was largest at topographical sites particularly vulnerable in the Cup model (e.g., corpus callosum, caudate-putamen, or deep cortical layers; Clarner et al., 2012). The formation of endogenous myelin-specific T cells appeared to be essential for this process, because animals solely "immunized" with CFA and PTX (lacking the MOG peptide) did not show significant peripheral cell recruitment. Subsequent immunohistochemical studies using anti-ionized calcium-binding adapter molecule 1 (Iba1) and anti-CD3 displayed the recruitment of monocytes and lymphocytes (Fig. 1D).

Peripheral leukocyte infiltration into the brain is tightly regulated at the level of the postcapillary venules and involves two distinct steps: (1) transmigration across the vascular wall into the perivascular space (i.e., Virchow-Robin space); and (2) progression through the glia limitans perivascularis (Rezai-Zadeh et al., 2009). A more detailed characterization of infiltrates revealed that, based on the distribution of inflammatory cells, two distinct lesion types could be discriminated: (1) lesions with inflammatory cells residing within the perivascular spaces (Fig. 1E1); and (2) lesions with inflammatory cells penetrating into the surrounding brain parenchyma (Fig. 1E2). As shown in Figure 1, F1 and F2, parenchymal immune cell invasion was paralleled by significant demyelination. Beyond, anti-Glial fibrillary acidic protein (GFAP) and anti-aquaporin-4 (AQP4) stains revealed a morphologically and functionally disrupted glia limitans perivascularis in those lesions with immune cell invasion into the brain parenchyma (Fig. 1G,H). The barrier of $\mathrm{GFAP}^{+}$astrocyte foot processes was discontinuous (Fig. 1G2), and AQP4 was found to be focally absent (Fig. 1H2). Furthermore, we quantified the number of parenchymal $\mathrm{CD}^{+}{ }^{+}$lymphocytes and found higher numbers in Cup-MOG animals (Fig. 1I). In support of this notion, gene expression analysis of a separate cohort of animals displayed high forebrain expression of the T-cell receptors $\mathrm{T} 3$ delta (CD3d) and $\varepsilon$ (CD3e) chain in Cup-MOG animals. Expression of T-cell receptor-CD3 complex components were only detected in minute amounts in all other groups (data not shown). A consistent MS feature is the transient loss of vascular impermeability (Cotton et al., 2003). Therefore, we tested whether observed histopathological alterations are transient or permanent. To this end, telencephalic lesions were induced as described above. However, brains were analyzed 6 weeks after immunization instead at the peak of disease. As shown in Figure $1 \mathrm{~J}$, antiGFAP stains showed a continuous layer of astrocyte end feet around vessels at this later time point. Furthermore, anti-AQP4 stains did not show any signs of focal loss of immunoreactivity. We next were interested whether a temporal correlation exists between Cup-induced neurodegeneration and the phenomenon of peripheral immune cell recruitment. To this end, animals were fed Cup for 3 weeks. However, in contrast to the already described experiments, MOG immunization was performed 4 instead of 2 weeks after cessation of the Cup intoxication. As shown in Figure $1 K$, immune cell recruitment was significantly lower under this experimental setting.

So far, we were able to demonstrate that peripheral encephalitogenic immune cells invade the forebrain parenchyma in case a neurodegenerative trigger is present before MOG immunization is performed. As shown in Figure 2A, ORO-stained sections showed a significant lipid increase in cervical lymph nodes of 


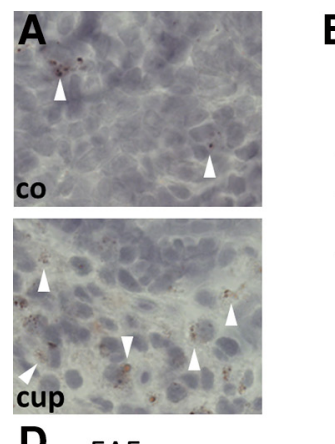

B

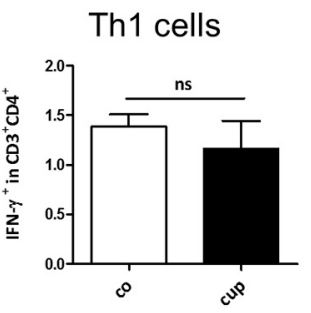

D $E A E$

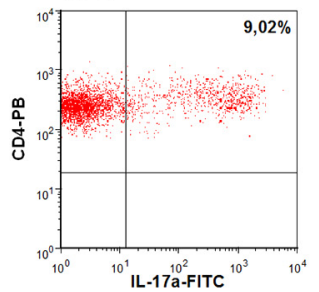

CUp/MOG

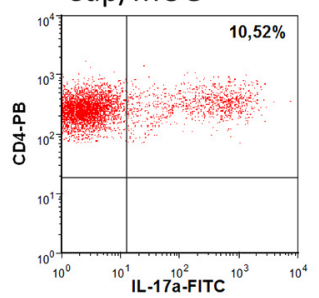

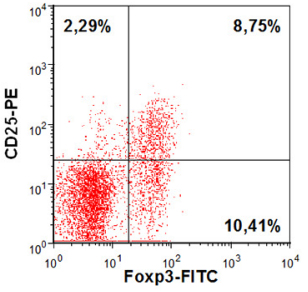
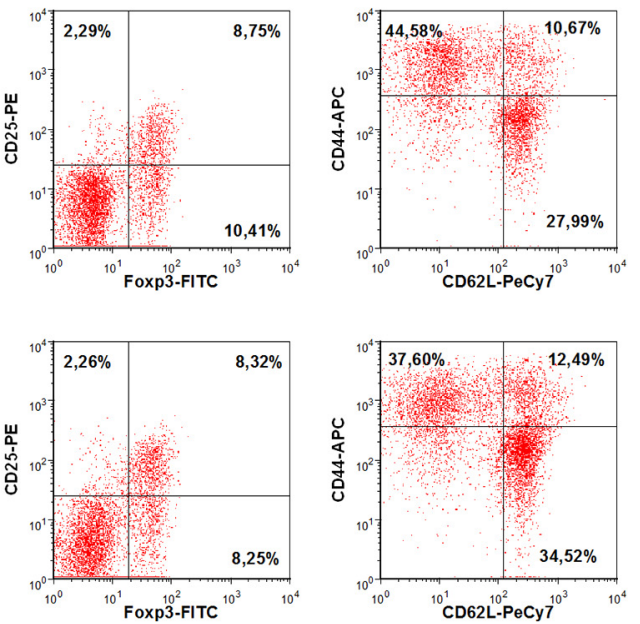

Th17 cells
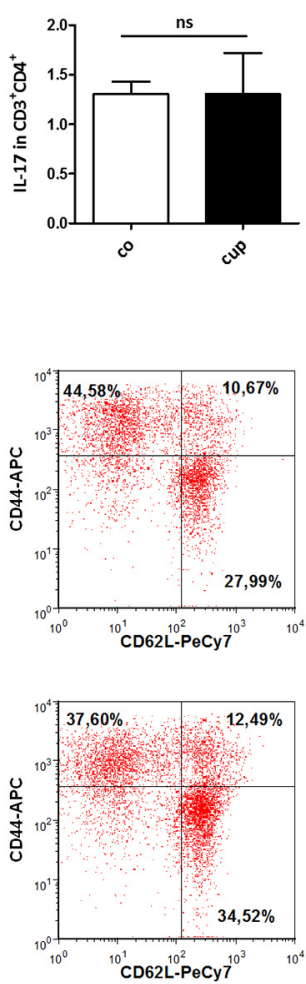

C
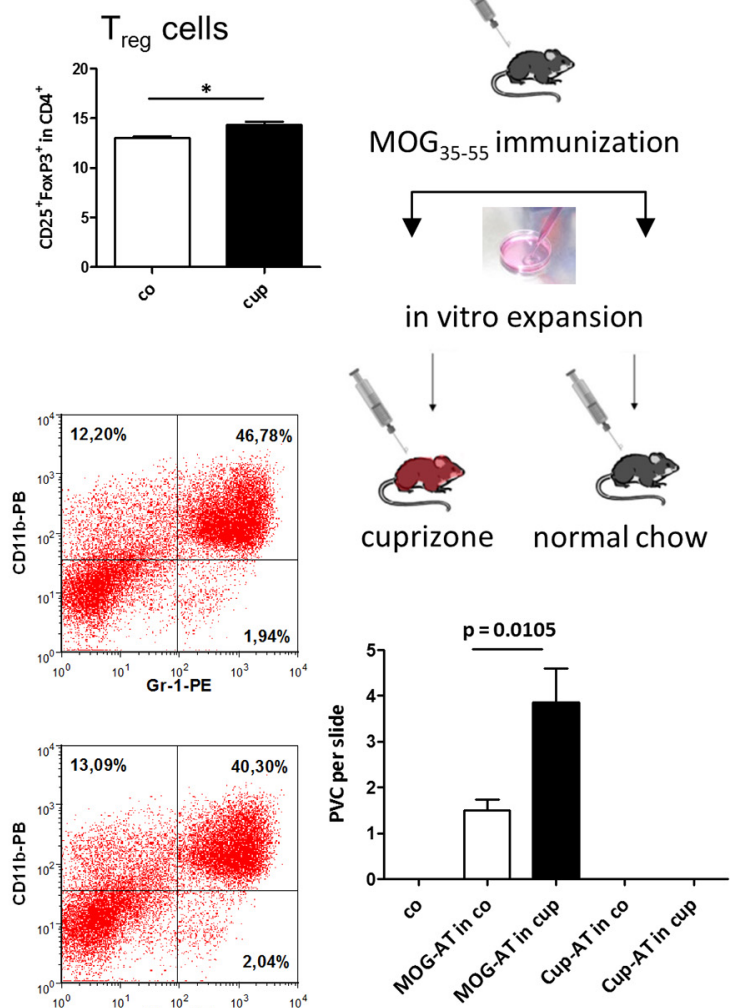

Figure 2. A, Representative ORO-stained cervical lymph nodes. White arrowheads highlight lipid-laden macrophages. $B$ shows relative numbers of $\mathrm{Th}_{1}, \mathrm{Th}_{17}$, and $\mathrm{T}_{\text {reg }}$ cells in cervical lymph nodes before MOG immunization. Note that the immune cell repertoire is not altered as a result of the Cup intoxication. C shows the number of forebrain perivascular infiltrates in different treatment groups. Note that perivascular infiltrates were virtually absent in co animals $(i)$ and in animals in which cultured and stimulated splenocytes, isolated from Cup-exposed animals, were transferred into either co animals (Cup-AT in co) or Cup-intoxicated animals (Cup-AT in cup; ii). In contrast, numerous perivascular infiltrates were present in classical AT experiments. Most importantly, the number of forebrain perivascular infiltrates is greater in animals with Cup-induced neurodegeneration before T-cell transfer (MOG-AT in co vs MOG-AT in cup). PVC, Perivascular cuffs. D, Immune cell

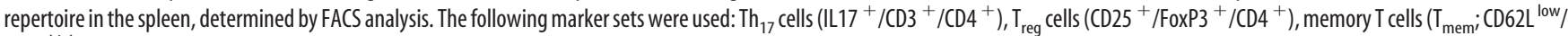
CD44 $\left.{ }^{\text {high }} / \mathrm{CD} 4^{+}\right)$, and myeloid-derived suppressor cells $\left(\mathrm{CD} 11 \mathrm{~b}^{+} / \mathrm{Gr} 1^{+}\right)$.

Cup-treated animals, indicating transfer of myelin material after Cup-induced neurodegeneration. Although we did not find an altered T-cell phenotype in these lymph nodes (Fig. 2B), peripheral immune cell recruitment in our novel combinatory model might occur as a consequence of released myelin debris during the Cup intoxication period that is transported to cervical lymph nodes. If present there in relevant amounts, local myelin antigens might influence the immune cell repertoire during MOG immunization. To check for this possibility, we first performed AT of in vitro MOG-reactivated lymphocytes. To this end, donor mice were immunized with MOG, killed at the first day of clinical signs, and their spleens harvested. Clonal expansion of MOGreactive lymphocytes was accomplished in vitro, and 20-30 million cells were injected into mice with or without previous Cup-induced neurodegeneration (for experimental setup, see Fig. 2C). Cup-pretreated animals displayed the tendency of a more delayed disease onset (AT, $9.2 \pm 0.73$ vs Cup-AT, $10.8 \pm$ $0.49 \mathrm{~d}$ after adoptive cell transfer; $p=0.11$ ), although this difference was not significant. Notably, mice fed Cup before adoptive T-cell transfer (MOG-AT in cup) showed a higher number of perivascular infiltrates compared with animals without previous Cup feeding (MOG-AT in co; Fig. 2C). It should also be stipulated that, if the same procedure was performed with splenocytes isolated from Cup-treated animals, recipient mice, regardless of being pre-intoxicated with Cup (Cup-AT in cup) or not (Cup-AT in co), did not develop any clinical signs of EAE. This indicates that no relevant numbers of autoreactive $\mathrm{T}$ cells are generated in the Cup model. Furthermore, we analyzed the immune cell signature in the spleen by means of flow cytometry. As demonstrated in Figure $2 \mathrm{D}$, numbers of T-helper $17\left(\mathrm{Th}_{17}\right)$ cells $\left(\mathrm{IL}_{17}{ }^{+} / \mathrm{CD}^{+} / \mathrm{CD}^{+}\right), \mathrm{T}_{\text {reg }}$ cells $\left(\mathrm{CD} 25^{+} / \mathrm{FoxP}^{+} / \mathrm{CD}^{+}\right)$, memory $\mathrm{T}$ cells $\left(\mathrm{CD} 62 \mathrm{~L}^{\text {low }} / \mathrm{CD} 44^{\text {high }} / \mathrm{CD} 4^{+}\right)$, and myeloidderived suppressor cells $\left(\mathrm{CD} 11 \mathrm{~b}^{+} / \mathrm{Gr}^{+}\right)$were induced comparably. Mean percentage of $\mathrm{Th}_{17}$ cells in $\mathrm{CD} 3^{+} / \mathrm{CD} 4^{+}$ lymphocytes were $12.4 \pm 2.7 \%$ in EAE versus $8.4 \pm 1.2 \%$ in Cup-MOG animals $(p=0.24)$, and mean percentage of $\mathrm{T}_{\text {reg }}$ cells in $\mathrm{CD}^{+}{ }^{+}$lymphocytes were $8.1 \pm 0.4 \%$ in EAE versus $8.4 \pm 0.5 \%$ in Cup-MOG animals $(p=0.69)$. Comparable results were obtained in isolated cervical lymph node cells (data not shown). Furthermore, absolute cell numbers, total monocytes (F4-80 cells), and neutrophils were induced to a comparable extent in the blood of either treatment group (data not shown).

\section{Discussion}

We investigated possible interactions between the injured forebrain parenchyma and leukocytes after Cup-induced neurodegeneration. Here, we demonstrate that neurodegenerative processes within the forebrain are at the beginning of a signaling cascade, which finally results in the recruitment of peripheral leukocytes and in consequence in the formation of a new, inflammatory, demyelinating lesion. The neurodegen- 
erative process was induced by Cup feeding of adult mice for 3 weeks.

Two important aspects need to be discussed with regard to the relevance of our findings for MS pathogenesis. First, can neurodegeneration trigger a subsequent peripheral autoreactive immune response that finally leads to lesion formation as seen in MS? Second, is MS a disease where primary neurodegeneration triggers immune cell recruitment to the sites of pathology? With respect to the first question, we found no clear-cut evidence that autoreactive $\mathrm{T}$ cells are generated in the Cup model (Fig. 2). The numbers of $\mathrm{Th}_{1}$ and $T \mathrm{~T}_{17}$ cells in peripheral lymphoid organs are unaltered, and the transfer of isolated splenocytes from Cupexposed animals to recipient mice does not result in immunemediated CNS inflammation. These results are in line with previous data presented by Locatelli et al. (2012). They showed that, even in a strong proinflammatory experimental setting, myelin antigens in CNS-draining lymph nodes failed to initiate antiCNS immunity. Thus, our study does not support the concept that anti-CNS immunity is attributable to a primary neurodegenerative event. Concerning the second question, our data suggest that encephalitogenic $\mathrm{T}$ cells together with monocytes are recruited to neurodegenerative lesion sites. The fact that neurodegenerative events can trigger the recruitment of peripheral immune cells into the CNS has been shown in experimental paradigms of peripheral facial nerve injury (Raivich et al., 1998), cortical cryoinjury, or eyeball enucleation (Konno et al., 1990). We are the first to describe such a mechanism in an MS-relevant context. It was our aim to model early MS-related degenerative processes as closely as possible and therefore used the Cup model that harbors several characteristics of early MS lesions. For example, careful pathological examinations have revealed that the earliest myelin abnormalities begin at the inner myelin sheath that surrounds still-myelinated axons (Rodriguez and Scheithauer, 1994; Aboul-Enein et al., 2003). Such alterations can also be found in the Cup model (Ludwin and Johnson, 1981). Furthermore, early MS lesions are characterized by selective oligodendrocyte degeneration with focal microglia activation (De Groot et al., 2001; Barnett and Prineas, 2004). Both aspects are characteristic for early Cup-induced lesions (Clarner et al., 2015).

Although the influx of peripheral immune cells into the CNS is a well known phenomenon in MS, the initial stages of this process are not well understood. To initiate CNS inflammation, encephalitogenic $\mathrm{T}$ cells must be activated in the periphery, gain access to the CNS, and then be reactivated by local antigen presenting cells, which present CNS antigen. The anatomy of the CNS poses unique challenges to this process because it is protected from cellular infiltration by specific borders, such as the blood-brain barrier. Although this study, for obvious reasons, does not answer the question of which factors contribute to encephalitogenic T-cell activation in the periphery, we clearly show that neurodegeneration orchestrates the important second step of MS lesion development, i.e., immune cell recruitment. In the absence of brain pathology, endothelial cells of the brain do not express the adhesion molecules that are necessary for activated $\mathrm{T}$ cells to adhere to the vessel wall. It is well known that astrocytes are activated in the Cup model (Kipp et al., 2011), and astrogliosis has often been thought to contribute to lesion formation, for example, by the secretion of chemokines. Because chemokine expression is induced in the Cup model (Clarner et al., 2015), we consider it likely that the Cup-induced brain pathology activates endothelial cells and subsequently facilitates the transmigration of $\mathrm{T}$ cells across the blood-brain barrier. An important question is how many of the recruited $\mathrm{T}$ cells to the Cup-induced lesion sites are autoreactive in this model and whether these are reactivated once they have entered the brain. The precise identity of the antigen-presenting cells responsible for reactivating $\mathrm{CD} 4{ }^{+}$ $\mathrm{T}$ cells within the CNS is not known, but microglia are potential candidates, especially at the onset of an inflammatory attack (Almolda et al., 2010). Because microglia activation is another cardinal feature of Cup-induced pathology, these cells might reactivate $\mathrm{T}$ cells at lesion sites in the presented model.

Our findings clearly have direct clinical relevance. Most approved MS drugs mainly harbor a peripheral mode of action. If lesion development starts with an initial neurodegenerative process, neuroprotective therapies might not just ameliorate the neurodegenerative but also the inflammatory aspect of the disease. A better understanding of factors regulating neurodegeneration-induced immune cell recruitment will pave the way for the development of novel therapeutic treatment strategies.

\section{References}

Aboul-Enein F, Rauschka H, Kornek B, Stadelmann C, Stefferl A, Brück W, Lucchinetti C, Schmidbauer M, Jellinger K, Lassmann H (2003) Preferential loss of myelin-associated glycoprotein reflects hypoxia-like white matter damage in stroke and inflammatory brain diseases. J Neuropathol Exp Neurol 62:25-33. Medline

Almolda B, González B, Castellano B (2010) Activated microglial cells acquire an immature dendritic cell phenotype and may terminate the immune response in an acute model of EAE. J Neuroimmunol 223:39-54. CrossRef Medline

Barnett MH, Prineas JW (2004) Relapsing and remitting multiple sclerosis: pathology of the newly forming lesion. Ann Neurol 55:458-468. CrossRef Medline

Baxi EG, DeBruin J, Tosi DM, Grishkan IV, Smith MD, Kirby LA, Strasburger HJ, Fairchild AN, Calabresi PA, Gocke AR (2015) Transfer of myelinreactive th17 cells impairs endogenous remyelination in the central nervous system of cuprizone-fed mice. J Neurosci 35:8626-8639. CrossRef Medline

Clarner T, Diederichs F, Berger K, Denecke B, Gan L, van der Valk P, Beyer C, Amor S, Kipp M (2012) Myelin debris regulates inflammatory responses in an experimental demyelination animal model and multiple sclerosis lesions. Glia 60:1468-1480. CrossRef Medline

Clarner T, Janssen K, Nellessen L, Stangel M, Skripuletz T, Krauspe B, Hess FM, Denecke B, Beutner C, Linnartz-Gerlach B, Neumann H, Vallières L, Amor S, Ohl K, Tenbrock K, Beyer C, Kipp M (2015) CXCL10 triggers early microglial activation in the cuprizone model. J Immunol 194: 3400-3413. CrossRef Medline

Coles AJ, Wing MG, Molyneux P, Paolillo A, Davie CM, Hale G, Miller D, Waldmann H, Compston A (1999) Monoclonal antibody treatment exposes three mechanisms underlying the clinical course of multiple sclerosis. Ann Neurol 46:296-304. CrossRef Medline

Cotton F, Weiner HL, Jolesz FA, Guttmann CR (2003) MRI contrast uptake in new lesions in relapsing-remitting MS followed at weekly intervals. Neurology 60:640-646. CrossRef Medline

De Groot CJ, Bergers E, Kamphorst W, Ravid R, Polman CH, Barkhof F, van der Valk P (2001) Post-mortem MRI-guided sampling of multiple sclerosis brain lesions: increased yield of active demyelinating and (p)reactive lesions. Brain 124:1635-1645. CrossRef Medline

Doan V, Kleindienst AM, McMahon EJ, Long BR, Matsushima GK, Taylor LC (2013) Abbreviated exposure to cuprizone is sufficient to induce demyelination and oligodendrocyte loss. J Neurosci Res 91:363-373. CrossRef Medline

Grosse-Veldmann R, Becker B, Amor S, van der Valk P, Beyer C, Kipp M (2015) Lesion expansion in experimental demyelination animal models and multiple sclerosis lesions. Mol Neurobiol. Advance online publication. Retrieved December 26, 2016. doi:10.1007/s12035-015-9420-y. CrossRef Medline

Kipp M, Clarner T, Dang J, Copray S, Beyer C (2009) The cuprizone animal model: new insights into an old story. Acta Neuropathol 118:723-736. CrossRef Medline

Kipp M, Gingele S, Pott F, Clarner T, van der Valk P, Denecke B, Gan L, Siffrin V, Zipp F, Dreher W, Baumgartner W, Pfeifenbring S, Godbout R, Amor 
S, Beyer C (2011) BLBP-expression in astrocytes during experimental demyelination and in human multiple sclerosis lesions. Brain Behav Immun 25:1554-1568. CrossRef Medline

Konno H, Yamamoto T, Suzuki H, Yamamoto H, Iwasaki Y, Ohara Y, Terunuma H, Harata N (1990) Targeting of adoptively transferred experimental allergic encephalitis lesion at the sites of wallerian degeneration. Acta Neuropathol 80:521-526. CrossRef Medline

Kremenchutzky M, Rice GP, Baskerville J, Wingerchuk DM, Ebers GC (2006) The natural history of multiple sclerosis: a geographically based study 9: observations on the progressive phase of the disease. Brain 129: 584-594. CrossRef Medline

Locatelli G, Wörtge S, Buch T, Ingold B, Frommer F, Sobottka B, Krüger M, Karram K, Bühlmann C, Bechmann I, Heppner FL, Waisman A, Becher B (2012) Primary oligodendrocyte death does not elicit anti-CNS immunity. Nat Neurosci 15:543-550. CrossRef Medline

Lucchinetti C, Brück W, Parisi J, Scheithauer B, Rodriguez M, Lassmann H (2000) Heterogeneity of multiple sclerosis lesions: implications for the pathogenesis of demyelination. Ann Neurol 47:707-717. CrossRef Medline

Ludwin SK, Johnson ES (1981) Evidence for a "dying-back" gliopathy in demyelinating disease. Ann Neurol 9:301-305. CrossRef Medline

Maggi P, Macri SM, Gaitán MI, Leibovitch E, Wholer JE, Knight HL, Ellis M, Wu T, Silva AC, Massacesi L, Jacobson S, Westmoreland S, Reich DS (2014) The formation of inflammatory demyelinated lesions in cerebral white matter. Ann Neurol 76:594-608. CrossRef Medline

Maña P, Fordham SA, Staykova MA, Correcha M, Silva D, Willenborg DO,
Liñares D (2009) Demyelination caused by the copper chelator cuprizone halts $\mathrm{T}$ cell mediated autoimmune neuroinflammation. J Neuroimmunol 210:13-21. CrossRef Medline

Raivich G, Jones LL, Kloss CU, Werner A, Neumann H, Kreutzberg GW (1998) Immune surveillance in the injured nervous system: T-lymphocytes invade the axotomized mouse facial motor nucleus and aggregate around sites of neuronal degeneration. J Neurosci 18:5804-5816. Medline

Rezai-Zadeh K, Gate D, Town T (2009) CNS infiltration of peripheral immune cells: D-Day for neurodegenerative disease? J Neuroimmune Pharmacol 4:462-475. CrossRef Medline

Rice GP, Filippi M, Comi G (2000) Cladribine and progressive MS: clinical and MRI outcomes of a multicenter controlled trial. Cladribine MRI Study Group. Neurology 54:1145-1155. CrossRef Medline

Rodriguez M, Scheithauer B (1994) Ultrastructure of multiple sclerosis. Ultrastruct Pathol 18:3-13. CrossRef Medline

Scalfari A, Neuhaus A, Degenhardt A, Rice GP, Muraro PA, Daumer M, Ebers GC (2010) The natural history of multiple sclerosis: a geographically based study 10: relapses and long-term disability. Brain 133:1914-1929. CrossRef Medline

Slowik A, Schmidt T, Beyer C, Amor S, Clarner T, Kipp M (2015) The sphingosine 1-phosphate receptor agonist FTY720 is neuroprotective after cuprizone-induced CNS demyelination. Br J Pharmacol 172:80-92. CrossRef Medline

Stys PK, Zamponi GW, van Minnen J, Geurts JJ (2012) Will the real multiple sclerosis please stand up? Nat Rev Neurosci 13:507-514. CrossRef Medline 